\title{
Assessment of dynamic structural instabilities across 24 cubic inorganic halide perovskites
}

DOI:

$10.1063 / 1.5131575$

\section{Document Version}

Accepted author manuscript

Link to publication record in Manchester Research Explorer

\section{Citation for published version (APA):}

Yang, R. X., Skelton, J. M., Da Silva, E. L., Frost, J. M., \& Walsh, A. (2020). Assessment of dynamic structural instabilities across 24 cubic inorganic halide perovskites. The Journal of chemical physics, 152(2), 024703. https://doi.org/10.1063/1.5131575

\section{Published in:}

The Journal of chemical physics

\section{Citing this paper}

Please note that where the full-text provided on Manchester Research Explorer is the Author Accepted Manuscript or Proof version this may differ from the final Published version. If citing, it is advised that you check and use the publisher's definitive version.

\section{General rights}

Copyright and moral rights for the publications made accessible in the Research Explorer are retained by the authors and/or other copyright owners and it is a condition of accessing publications that users recognise and abide by the legal requirements associated with these rights.

\section{Takedown policy}

If you believe that this document breaches copyright please refer to the University of Manchester's Takedown Procedures [http://man.ac.uk/04Y6Bo] or contact uml.scholarlycommunications@manchester.ac.uk providing relevant details, so we can investigate your claim.

\section{OPEN ACCESS}




\title{
Assessment of dynamic structural instabilities across 24 cubic inorganic halide perovskites
}

\author{
Ruo Xi Yang, ${ }^{1,2}$ Jonathan M. Skelton, ${ }^{3}$ Estelina L. da Silva, ${ }^{4}$ Jarvist M. Frost, ${ }^{5}$ and Aron Walsh ${ }^{1,6, a)}$ \\ ${ }^{1)}$ Department of Materials, Imperial College London, Exhibition Road, London SWr $2 A Z$, \\ $U K$ \\ ${ }^{2)}$ Molecular Foundry, Lawrence Berkeley National Laboratory, 1 Cyclotron Rd, Berkeley, CA 94720, \\ USA \\ ${ }^{3)}$ Department of Chemistry, University of Manchester, Manchester M13 9PL, \\ $U K$ \\ ${ }^{4)}$ Instituto de Diseño para la Fabricación y Producción Automatizada, MALTA Consolider Team, \\ Universitat Politècnica de València, València, Spain, 46022 Valencia, Spain \\ ${ }^{5)}$ Department of Physics, Imperial College London, Exhibition Road, London SW7 $2 A Z$, \\ $U K$ \\ ${ }^{6)}$ Department of Materials Science and Engineering, Yonsei University, Seoul 120-749, \\ Korea
}

(Dated: 27 January 2020)

\begin{abstract}
Metal halide perovskites are promising candidates for next-generation photovoltaic and optoelectronic applications. The flexible nature of the octahedral network introduces complexity when understanding their physical behavior. It has been shown that these materials are prone to decomposition, phase competition, and the local crystal structure often deviates from the average space group symmetry. To make stable phase-pure perovskites, understanding their structure-composition relations is of central importance. We demonstrate, from lattice dynamics calculations, that the 24 inorganic perovskites $\mathrm{ABX}_{3}(\mathrm{~A}=\mathrm{Cs}, \mathrm{Rb} ; \mathrm{B}=\mathrm{Ge}, \mathrm{Sn}, \mathrm{Pb} ; \mathrm{X}=$ $\mathrm{F}, \mathrm{Cl}, \mathrm{Br}, \mathrm{I})$ exhibit instabilities in their cubic phase. These instabilities include cation displacements, octahedral tilting, and Jahn-Teller distortions. The magnitudes of the instabilities vary depending on the chemical identity and ionic radii of the composition.The tilting instabilities are energetically dominant, and reduce as the tolerance factor increases, whereas cation displacements and Jahn-Teller type distortions depend on the interactions between the constituent ions. We further considered representative tetragonal, orthorhombic and monoclinic perovskites phases to obtain phonon-stable phases for each composition. This work provides insights into the thermodynamic driving force of the instabilities and will help guide synthesis in material screening.
\end{abstract}

\section{INTRODUCTION}

Since the discovery of photoconductivity in the caesium lead halides $\left(\mathrm{CsPbX}_{3}[\mathrm{X}=\mathrm{Cl}, \mathrm{Br}, \mathrm{I}]\right)^{1}$, the semiconducting properties of halide perovskites have attracted significant research attention, including analogous compounds based on $\mathrm{Sn}$ and $\mathrm{Ge}^{2-4}$ Interest has since expanded to the hybrid organic-inorganic perovskites with potential applications including field-effect transistors, ${ }^{5}$ photovoltaics, ${ }^{6,7}$ and light-emitting diodes. ${ }^{8}$ This family of materials display a unique combination of physical and chemical properties, including fast ion and electron transport, ${ }^{9-11}$ long carrier diffusion lengths, ${ }^{12}$ and high quantum efficiency. ${ }^{13}$

The crystallography of lead halide perovskites dates back to the $1950 \mathrm{~s}$, where the high-temperature crystal structures of the $\mathrm{CsPbX}_{3}$ series were determined to be the archetypal cubic perovskite structure (space group $P m \overline{3} m)$. The same structure was later reported for the $\mathrm{CH}_{3} \mathrm{NH}_{3} \mathrm{PbX}_{3}$ series. ${ }^{14}$ In all cases, phase transitions to lower symmetry perovskite phases are observed as the temperature is reduced, e.g. in $\mathrm{CsPbCl}_{3}$ there is a tran-

\footnotetext{
a)Electronic mail: a.walsh@imperial.ac.uk
}

sition to a tetragonal phase at $320 \mathrm{~K}$, an othorhombic phase at $316 \mathrm{~K}$, and a monoclinic phase at $310 \mathrm{~K} .{ }^{15}$

The nature of the high temperature cubic phase of the halide perovskites has received less attention. Analysis of the X-ray pair distribution functions of $\mathrm{CH}_{3} \mathrm{NH}_{3} \mathrm{SnBr}_{3}$ suggested that the the local cubic symmetry was broken with significant distortions of the corner-sharing octahedral network. ${ }^{16}$ It was recently confirmed, from both inelastic X-ray and neutron total scattering that the cubic phase of $\mathrm{CH}_{3} \mathrm{NH}_{3} \mathrm{PbI}_{3}$ is also symmetry broken. ${ }^{17-19}$ These observations have been associated with rotational disorder of the molecular $\mathrm{CH}_{3} \mathrm{NH}_{3}{ }^{+}$cation.

Relatively little work has been reported on the inorganic halide perovskite counterparts. It was shown by Xray total scattering that $\mathrm{CsPbX}_{3}$ nanocrystals always exhibit orthorhombic tilting of the octahedra within locallyordered subdomains. ${ }^{20}$ Furthermore, low-frequency Raman scattering has confirmed that $\mathrm{CsPbBr}_{3}$ undergoes dynamical polar fluctuations at $300 \mathrm{~K}$, even though analysis of XRD measurement suggests the structure to be cubic at that temperature. ${ }^{21}$ Evidence of symmetry breaking has also been reported from analysis of the temperature-dependant photoluminescence of Cs and $\mathrm{CH}_{3} \mathrm{NH}_{3}$ compounds. ${ }^{22}$

In this work, we demonstrate that vibrational instabilities are common to inorganic halide perovskites in the 
$\mathrm{ABX}_{3}(\mathrm{~A}=\mathrm{Cs}, \mathrm{Rb} ; \mathrm{B}=\mathrm{Ge}, \mathrm{Sn}, \mathrm{Pb} ; \mathrm{X}=\mathrm{F}, \mathrm{Cl}, \mathrm{Br}, \mathrm{I})$ family. The associated dynamic disorder is a consequence of the flexibility associated with the corner-sharing network of inorganic octahedra, which includes rigid-unit tilting modes, distortions of the octahedra, and cation displacements. Through first-principles lattice-dynamics calculations, we assess the chemical and thermodynamic driving forces for these instabilities and discuss the consequences for the material properties.

\section{CLASSIFICATION OF STRUCTURAL INSTABILITIES}

The aristotype cubic perovskite structure is usually adopted at high temperature, while at low temperature an assortment of lower symmetry phases (e.g. tetragonal, orthorhombic, monoclinic, rhombohedral) are observed. The lattice distortions associated with these transitions can be divided into three categories: (i) polar displacement of the A or B cations away from their high symmetry positions; (ii) rigid tilting of the corner-sharing $\mathrm{BX}_{6}$ octahedra; and (iii) collective Jahn-Teller distortion of the $\mathrm{BX}_{6}$ octahedra. ${ }^{23-26}$

Cation displacements are usually responsible for socalled proper ferroelectricity, which is associated with the presence of a soft polar phonon mode at the $\Gamma$ point in the phonon Brillouin Zone. For example, $\mathrm{BaTiO}_{3}$ and $\mathrm{PbTiO}_{3}$ exhibit spontaneous polarization due to the displacement of the Ti atom from the center of its octahedron. ${ }^{27}$ The displacive phonon mode is at the Brillouin Zone center which implies an in-phase periodic distortion across the crystal and thus a macroscopic polarization.

On the other hand, octahedral tilting due to zoneboundary phonon modes (e.g. at the $M\left(\frac{1}{2}, \frac{1}{2}, 0\right)$ or $R\left(\frac{1}{2}, \frac{1}{2}, \frac{1}{2}\right)$ special points in the first Brillouin Zone of the cubic structure) result in an anti-phase periodic distortion. The opposite polarization generated in neighbouring unit cells cancels out and there is no resulting macroscopic polarization. Due to this, these are often referred to as antiferroelectric or antipolar distortions. In-phase tilting corresponds to an $M_{3}^{+}$mode denoted by + in Glazer's notation $a^{0} a^{0} c^{+}$, whereas out-of-phase tilting corresponds to an $R_{4}^{+}$mode denoted by a - sign in $a^{0} a^{0} c^{-} .^{28}$ A linear combination of both modes can define all the possible rigid tilting modes in the perovskite systems. ${ }^{24}$ We previously explored the behaviour of the $M_{3}^{+}$mode in the caesium lead and tin halides. ${ }^{29}$

A third type of distortion is due to the Jahn-Teller (JT) effect. The octahedra distort by elongation or shortening of the B-X bond, and sometimes by off-centering of the B cation. A first-order JT distortion is commonly observed in transition metals with degenerate electronic ground states such as $\mathrm{Cu}(\mathrm{II})$. Shortening/elongating the nearest-neighbor bonds and generating a crystal field lifts the degeneracy of the partially occupied $d$ band (e.g. $\mathrm{d}^{9}$ for $\mathrm{Cu}^{2+}$ in $\mathrm{KCuF}_{3}$ ) and allows for a lowering of the en- ergy. Cases such as $\mathrm{Pb}$ (II) $6 \mathrm{~s}^{2}$ where the structural distortion allows mixing with nominally empty $6 s^{0}$ orbitals are usually referred to as second-order JT distortions. ${ }^{30}$

These classes of distortion are not necessarily independent, but can couple via (anharmonic) interactons between phonon modes. Benedek et al. found that this coupling acts to suppress ferroelectricity in many oxide perovskites. ${ }^{25}$ Moreover, there has been large interest in designing new "hybrid improper" ferroelectricity through coupling between non-polar modes in Ruddlesden-Popper phase perovskites. ${ }^{31}$ In particular, tilting coupled with Jahn-Teller distortions (so-called "pseudo" rotations) are considered one route for designing multiferroic materials. ${ }^{32-34}$

\section{METHODS}

Model structures for 24 inorganic halide compounds $\mathrm{ABX}_{3}(\mathrm{~A}=\mathrm{Cs}, \mathrm{Rb} ; \mathrm{B}=\mathrm{Ge}, \mathrm{Sn}, \mathrm{Pb} ; \mathrm{X}=\mathrm{F}, \mathrm{Cl}$, $\mathrm{Br}, \mathrm{I})$ were taken from the Inorganic Crystal Structure Database where available, and the remaining generated by atomic substitution of similar structures. Density functional theory (DFT) as implemented in the pseudopotential plane-wave code $\operatorname{VASP}^{35,36}$ was used to optimize the crystal structures of four commonlyobserved phases viz. cubic Pm $\overline{3} m$, tetragonal $P 4 / \mathrm{mmm}$, orthorhombic Pnma and monoclinic $P 2_{1} / \mathrm{m}$. The structures were fully relaxed with the PBEsol exchangecorrelation functional. ${ }^{37-39}$ Explicit convergence testing identified a plane-wave kinetic-energy cutoff of $800 \mathrm{eV}$ and an electronic Brillouin zone sampling with an $8 \times 8 \times 8$ mesh for the cubic and orthorhombic phases, a $k$-point mesh of $6 \times 6 \times 8$ mesh for the tetragonal phases, and a $4 \times 4 \times 4$ mesh monoclinic phases to produce accurate phonon frequencies.

The formation enthalpy of each crystal can be calculated from

$$
\Delta H_{A B X_{3}}=E_{A B X_{3}}-\mu_{A}-\mu_{B}-3 \mu_{X}
$$

where $E_{A B X_{3}}$ is the total energy obtained in DFT calculation, $\mu$ are the chemical potentials of the constituent elements. When comparing the differences in $\Delta H_{A B X_{3}}$ between different phases with the same composition, the $\mu$ terms cancel and $\Delta H$ is simply the difference in DFT total energies for the different phases.

Harmonic phonon dispersion and density of states (DoS) curves were obtained using the finite displacement method implemented in the open-source PHONOPY package, with VASP used as the force calculator. For each phase, a series of symmetry-independent displacements were generated in $2 \times 2 \times 2$ supercell expansions, chosen to be commensurate with the zone-boundary symmetry points, to obtain the force-constant matrices, which were then used to obtain phonon frequencies and eigenvectors at arbitrary phonon wavevectors q.

To map out the anharmonic potential energy surfaces associated with the phonon instabilities, we use the open- 
a
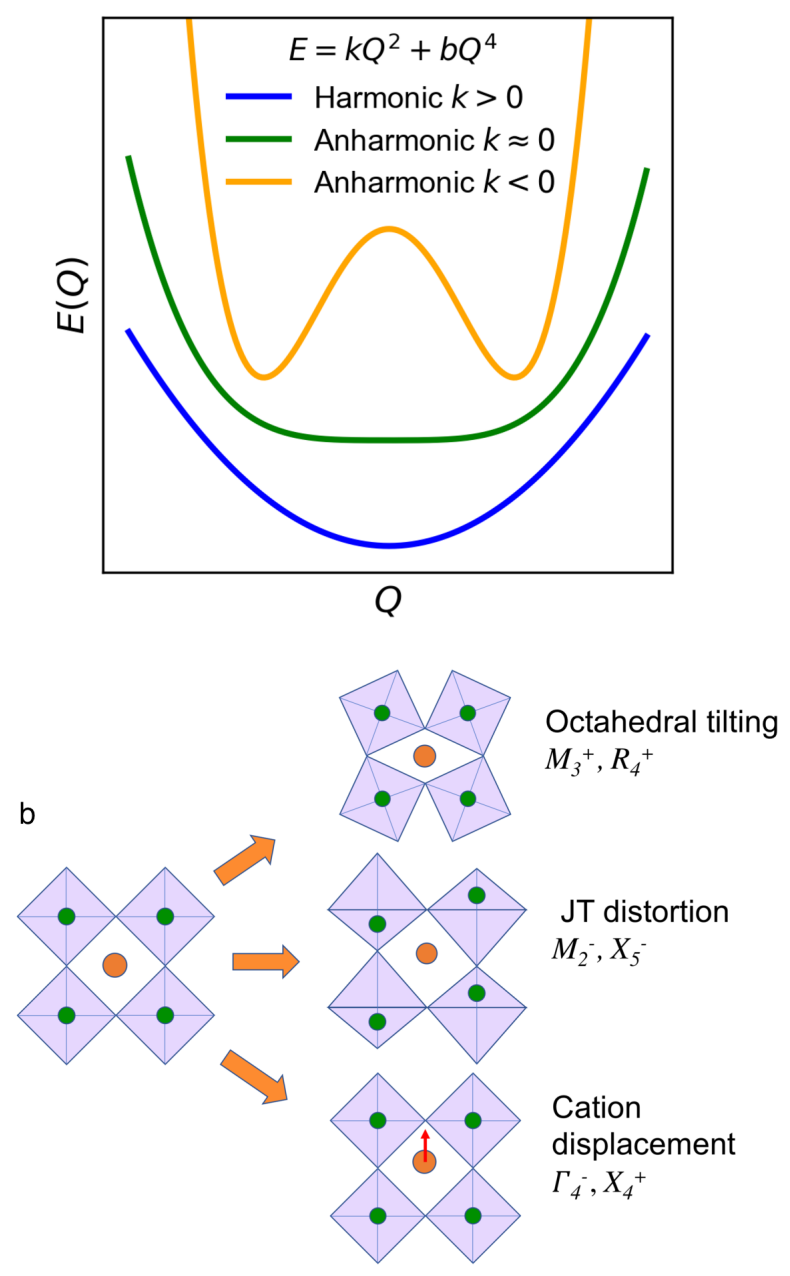

FIG. 1. a) Schematic potential energy surface along harmonic and anharmonic phonon modes as a function of the phonon normal mode coordinate $Q$. b) Schematic illustration of the three classes of distortion observed in cubic perovskites and the associated irreducible representations from group theory. The ferroelectric displacement is associated with a zone-centre phonon mode that leads to macroscopic electric polarization. The octahedral tilting is associated with a zone-boundary phonon mode and results in an expansion of the unit cell. Jahn-Teller distortions can be associated with either a zonecentre or zone-boundary mode, but in the cubic perovskites studied here manifest as zone-boundary $\mathrm{M}$ or $\mathrm{X}$ modes. These distortions may coexist in real materials.

source ModeMaP package. ${ }^{40,41}$ A sequence of displaced structures in a commensurate supercell expansion are generated by displacing along the phonon eigenvectors over a range of amplitudes of the normal-mode coordinate $Q$, and the total energies of the "frozen phonon" structures are evaluated from single-point DFT calculations. The $E(Q)$ curves are then fitted to a polynomial function, with the number of terms depending on the form of the potential well. These anharmonic energy surfaces then effectively include higher-order terms in the potential energy as a function of the nuclear coordinates, in the basis of the harmonic eigenvectors.

\section{PHASE DIVERSITY OF INORGANIC HALIDE PEROVSKITES}

A search of the literature shows that only a fraction of the 24 chemical combinations studied here have been reported experimentally, as summarised in Table I. The majority of the inorganic halide perovskites are reported to adopt the cubic structure (space group $P m \overline{3} m$ ) at high temperature, including $\mathrm{CsGeCl}_{3}, \mathrm{CsPbCl}_{3}, \mathrm{CsPbBr}_{3}$, $\mathrm{CsPbI}_{3}, \mathrm{CsSnI}_{3}, \mathrm{RbGeI}_{3}, \mathrm{RbPbF}_{3},{ }^{42-48}$ while $\mathrm{CsSnBr}_{3}$ has been reported to be cubic at room temperature. ${ }^{2}$

The phase diversity can be qualitatively explained using the concept of the tolerance factor $\alpha$ introduced by Goldschmidt ${ }^{49}$ where:

$$
\alpha=\left(r_{A}+r_{X}\right) / \sqrt{2}\left(r_{B}+r_{X}\right)
$$

Values of $\alpha<1$ are usually associated with octahedral tilting due to an A cation that is smaller than the ideal value for the $\mathrm{BX}_{3}$ octahedral framework. This is the case for the majority of compounds considered here (see Table I), which explains the most stable phases being lower-symmetry space groups, typically Pnma, which is the ground state structure of $\mathrm{CsPbCl}_{3}, \mathrm{CsSnI}_{3}, \mathrm{RbPbF}_{3}$, $\mathrm{RbPbI}_{3} \cdot{ }^{43,45,46,48}$

However, many of the Ge compounds have $\alpha>1$, which often leads to B-site displacements and hence to polar structures due to loss of centrosymmetry. For instance, $\mathrm{CsGeCl}_{3}, \mathrm{CsGeBr}_{3}$ and $\mathrm{CsGeI}_{3}$ are reported to undergo an order-disorder phase transition from cubic to rhombehedral $(R 3 m)$ under ambient conditions. ${ }^{4,50}$

We computed the formation enthalpy and phonon dispersion of four phases for each composition (see Supporting Information). Imaginary frequencies (soft modes) present in the phonon dispersion indicate dynamic instabilities at $0 \mathrm{~K}$, whereas the absence of imaginary modes indicates the structure is a local potential-energy minimum. The stable phases of each composition are summarised in Table I. None are found to be most stable in the cubic or tetragonal phases, with the corresponding dispersion curves showing soft modes across the whole Brillouin zone, indicating multiple dynamical instabilities. Similarly, the tetragonal phases, derived from the cubic structure by in-phase octahedral rotation with Glazer notation $a^{0} a^{0} c^{+}$, also show multiple instabilities. This indicates that the tetragonal phases are intermediates formed on the symmetry lowering path from cubic to the lowest-energy symmetry-broken ground state.

The lower-symmetry phases of all compositions have more favourable formation enthalpies than the cubic phase, and the orthorhombic Pnma and monoclinic $P 2_{1} / m$ phase also have lower formation energies than tetragonal $P 4 / \mathrm{mmm}$ structure, showing that additional distortions lower the energy (Table I). This shows that 
the tetragonal phases are both dynamically and energetically unstable at $0 \mathrm{~K}$. However, the formation enthalpies of the Pnma and $P 2_{1} / m$ phases are similar, implying that the energy gain for further lowering the symmetry from orthorhombic to monoclinic is small. Consideration of the formation enthalpy differences can help to explain why the orthorhombic phase is the most commonly observed for halide perovskites.

\section{HARMONIC PHONON INSTABILITIES}

In the cubic perovskite phase, all compounds were found to exhibit imaginary modes at certain symmetry points in the phonon Brillouin zone (Table II). All compounds exhibit $M$-point instabilities. Apart from $\mathrm{CsSnBr}_{3}$, all the compounds also exhibit $\Gamma$-point instabilities. Perovskites other than $\mathrm{CsPbF}_{3}, \mathrm{CsSnBr}_{3}$ and $\mathrm{CsSnCl}_{3}$ also show $X$-point instabilities. In addition, $R$ point instabilities are found in all perovskites other than $\mathrm{CsGeX}_{3}(\mathrm{X}=\mathrm{F}, \mathrm{Cl}, \mathrm{Br})$ and $\mathrm{CsSnF}_{3}$. It is worth noting that all the $\mathrm{Rb}$ compounds feature instabilities at every high-symmetry point in the cubic perovskite Brillouin zone, in agreement with the fact that fewer $\mathrm{Rb}$ halide perovskites have been identified experimentally.

To provide a better understanding of the underlying chemical driving forces, we have categorized the instabilities into zone-centre cation displacements, zoneboundary octahedral tilting, and Jahn-Teller distortions (Fig. 1). We will show in the following sections how the chemistry of the compound determines the types of instabilities that occur.

\section{A. Octahedral tilting}

Instabilities corresponding to the octahedral tilting can be found at both the $M$ and $R$ points. Among the 24 cubic halide perovksites all show $M$-point instabilities, allowing comparisons to be made between the systems. Although the imaginary modes occur at the same reciprocal-space wavevector, they can correspond to different types of distortions in real space. The harmonic eigenvectors associated with these modes were therefore inspected to determine the distortion type. Some compounds have more than one $M$-point instability, but for simplicity we confine our discussion to the "most" imaginary phonon branch, i.e. the one lying lowest in the phonon dispersion, which is indicative of the largest negative curvature of the potential-energy surface around $Q$ $=0$.

$\mathrm{CsSnCl}_{3}, \quad \mathrm{CsSnBr}_{3}, \mathrm{CsSnI}_{3}, \quad \mathrm{CsPbX}_{3}, \mathrm{RbGeBr}_{3}$, $\mathrm{RbGeI}_{3}, \mathrm{RbSnCl}_{3}, \mathrm{RbSnBr}_{3}, \mathrm{RbSnI}_{3}$ and $\mathrm{RbPbX}_{3}$ display $M_{3}^{+}$instabilities corresponding to an in-phase octahedral tilting with Glazer notation $a^{0} a^{0} c^{+}$(Fig. 3). For $\mathrm{CsGeX}_{3}, \mathrm{CsSnF}_{3}, \mathrm{RbGeF}_{3}, \mathrm{RbGeCl}_{3}, \mathrm{RbSnF}_{3}$, the lowest-lying phonon mode corresponds to the $M_{2}^{-}$mode, which corresponds to a Jahn-Teller distortion and will be discussed in the following section.

Using the eigenvectors associated with the $M_{3}^{+}$mode, we mapped out the potential energy surface $E$ as a function of the normal-mode coordinate $Q$. The $E-Q$ curves for $M_{3}^{+}$in all compositions that adopt this instability are shown in Fig. 2. The resulting $E-Q$ surfaces correspond to symmetrical double-well potentials, with the central point $Q=0$ being the reference cubic phase. The well depth indicates the enthalpy gained by breaking the crystal symmetry. Shallow well depths $\left(\Delta E \approx k_{B} T\right)$ suggest that thermal energy can allow the system to explore parts of the potential close to $Q=0$. Such anharmonic double wells are common in perovskite structures ${ }^{59,60}$ and are characteristic of ferroelectric transitions for cases where $Q$ represents a polar distortion. ${ }^{61,62}$

The $\mathrm{Rb}$ compounds possess deeper minima than their Cs counterparts (Fig. 2), indicating a stronger energetic driving force for tilting. Within the $\mathrm{Rb}$ or $\mathrm{Cs}$ series, there are two distinct trends: (i) the double well depth increases monotonically when the B-site cation goes from $\mathrm{Ge} \rightarrow \mathrm{Sn} \rightarrow \mathrm{Pb}$; (ii) for compounds with the same B-site cation, the well depth increases as the $\mathrm{X}$ site is substituted from $\mathrm{F} \rightarrow \mathrm{Cl} \rightarrow \mathrm{Br} \rightarrow \mathrm{I}$.

Both phenomena can be interpreted by the geometry of the cubic perovskite crystal. Firstly, the Rb cation is too small for the octahedral cavity, which drives the collapse of the network towards the A cation. Secondly, the interplay between the size of the A-site cation and the volume of the cage also determines the magnitude of the tilting instability, i.e. the larger the ratio of the size of the cavity to the A-site cation, the stronger the tilting. This again follows the tolerance factor $\alpha$. If $\alpha$ is too small, the octahedral network will tend to tilt, as observed in $\mathrm{RbPbX}_{3}$, which have the smallest $\alpha(0.82$ -0.86 ) and the largest well depths. On the other hand, when $\alpha$ is close to or greater than one, such as in $\mathrm{CsGeX}_{3}$ (0.98-1.11), $\mathrm{RbGeF}_{3}$ (1.05) and $\mathrm{RbGeCl}_{3}(0.98)$, the $M_{3}^{+}$ tilting instability is not seen due to the limited space in the cuboctahedral cavity.

An almost-identical trend is observed for the out-ofphase tilting $R_{4}^{+}$mode, which suggests that the behavior is not limited to in-phase tilting but is common to all tilting modes.

\section{B. Zone-boundary Jahn-Teller distortion}

In addition to rigid octahedral tilting, some compounds show $M$-point imaginary frequencies associated with a different type of distortion. For most of the Ge perovskites and some of the Sn perovskites, including $\mathrm{CsGeF}_{3}, \mathrm{CsGeCl}_{3}, \mathrm{CsGeBr}_{3}, \mathrm{CsGeI}_{3}, \mathrm{RbGeF}_{3}$, $\mathrm{RbGeCl}_{3}, \mathrm{CsSnF}_{3}$, and $\mathrm{RbSnF}_{3}$, the imaginary modes with the steepest local curvature correspond to an $\mathrm{M}_{2}^{-}$ instability (Fig. 4). Instead of rigid rotation of the octahedra with fixed bond lengths, the $\mathrm{B}-\mathrm{X}$ bonds either shorten or lengthen and the B-site cation is displaced 
TABLE I. Difference in formation enthalpy $(\Delta H)$ for tetragonal $\left(P 4 / m b m, a^{0} a^{0} c^{+}\right)$, orthorhombic $\left(P n m a, a^{+} b^{-} b^{-}\right)$and monoclinic $\left(P 2_{1} / m, a^{+} b^{-} c^{-}\right)$phases of the $\mathrm{ABX}_{3}$ perovskites with respect to the cubic phase $\left(P m \overline{3} m, a^{0} a^{0} a^{0}\right)$. Phases identified as being phonon stable and experimentally-observed structures are also given. The tolerance factor is calculated using the Shannon ionic radii where available ${ }^{51}$, while the radius for $\operatorname{Sn}(\mathrm{II})(1.15 \AA)$ is taken from Ref. 52 .

\begin{tabular}{|c|c|c|c|c|c|c|}
\hline Composition & $\begin{array}{l}\Delta H(\mathrm{me} \\
P 4 / m b n\end{array}$ & $\begin{array}{l}\mathrm{J} / \text { formu } \\
\text { Pnma }\end{array}$ & $\begin{array}{l}P 2_{1} / m \\
\end{array}$ & Phonon stable phase & Experimentally observed & Tolerance factor \\
\hline $\mathrm{CsGeF}_{3}$ & -0.9 & -1 & -0.7 & & & 1.11 \\
\hline $\mathrm{CsGeCl}_{3}$ & -0.3 & -1 & -0.3 & & $P m \overline{3} m$ at $449 \mathrm{~K} / R 3 m$ at $\mathrm{RT}^{53}$ & 1.02 \\
\hline $\mathrm{CsGeBr}_{3}$ & -2.6 & -4.0 & -1.1 & & $R 3 m$ at $\mathrm{RT}^{4}$ & 1.01 \\
\hline $\mathrm{CsGeI}_{3}$ & -2.6 & -4.4 & -0.2 & & $R 3 m$ at $\mathrm{RT}^{4}$ & 0.98 \\
\hline $\mathrm{CsSnF}_{3}$ & 0.2 & -1.8 & -2.5 & & & 0.92 \\
\hline $\mathrm{CsSnCl}_{3}$ & -0.9 & -27.7 & -27.3 & & $P m \overline{3} m$ at $\mathrm{RT}^{54}$ & 0.88 \\
\hline $\mathrm{CsSnBr}_{3}$ & -22.8 & -35.9 & -35 & $P n m a$ and $P 2_{1} / m$ & $P m \overline{3} m$ at RT, Pnma at $100 \mathrm{~K}^{2,55}$ & 0.87 \\
\hline $\mathrm{CsSnI}_{3}$ & -634.1 & -692.9 & -691.8 & $P 2_{1} / m$ & $P m \overline{3} m$ at $500 \mathrm{~K} ; P n m a$ at $\mathrm{RT}^{46}$ & 0.86 \\
\hline $\mathrm{CsPbF}_{3}$ & -20.4 & -32 & -32 & $P n m a$ and $P 2_{1} / m$ & $P m \overline{3} m$ at $186 \mathrm{~K} ; R 3 c$ at $\mathrm{RT}^{56,57}$ & 0.90 \\
\hline $\mathrm{CsPbCl}_{3}$ & -63.8 & -85.5 & -85.3 & & $P m \overline{3} m$ at $320 \mathrm{~K} ; P n m a$ at $310 \mathrm{~K}^{15}$ & 0.87 \\
\hline $\mathrm{CsPbBr}_{3}$ & -72.5 & -100.5 & -100.4 & $P 2_{1} / m$ & $P m \overline{3} m$ at $403 \mathrm{~K} ; P n m a$ at $361 \mathrm{~K}^{58}$ & 0.86 \\
\hline $\mathrm{CsPbI}_{3}$ & -94.4 & -137.5 & -137.3 & Pnma & $P m \overline{3} m$ at $634 \mathrm{~K}^{45}$ & 0.85 \\
\hline $\mathrm{RbGeF}_{3}$ & -0.7 & -0.1 & -0.7 & & & 1.05 \\
\hline $\mathrm{RbGeCl}_{3}$ & -8.2 & -1.8 & -12.3 & & & 0.98 \\
\hline $\mathrm{RbGeBr}_{3}$ & -17.8 & -22.4 & -26.5 & & & 0.97 \\
\hline $\mathrm{RbGeI}_{3}$ & -41.3 & -47.4 & -65.2 & & $P m \overline{3} m$ at $533 \mathrm{~K}^{47}$ & 0.95 \\
\hline $\mathrm{RbSnF}_{3}$ & -73.2 & -97.9 & -97.9 & & & 0.87 \\
\hline $\mathrm{RbSnCl}_{3}$ & -105.4 & -156 & -155.8 & & & 0.84 \\
\hline $\mathrm{RbSnBr}_{3}$ & -103.7 & -158.2 & -157.7 & $P n m a$ and $P 2_{1} / m$ & & 0.84 \\
\hline $\mathrm{RbSnI}_{3}$ & -117.5 & -185.9 & -185.7 & $P n m a$ and $P 2_{1} / m$ & & 0.83 \\
\hline $\mathrm{RbPbF}_{3}$ & -134.9 & -188.4 & -188.3 & $P n m a$ and $P 2_{1} / m$ & $P m \overline{3} m$ at $515 \mathrm{~K} ; P n m a$ at $\mathrm{RT}^{48}$ & 0.86 \\
\hline $\mathrm{RbPbCl}_{3}$ & -170.4 & -249.2 & -196.7 & Pnma & & 0.83 \\
\hline $\mathrm{RbPbBr}_{3}$ & -171.3 & -255.2 & -197.1 & $P n m a$ and $P 2_{1} / m$ & & 0.83 \\
\hline $\mathrm{RbPbI}_{3}$ & -185.9 & -289.4 & -289.4 & $P n m a$ and $P 2_{1} / m$ & $P m \overline{3} m$ at $\mathrm{RT}^{45}$ & 0.82 \\
\hline
\end{tabular}
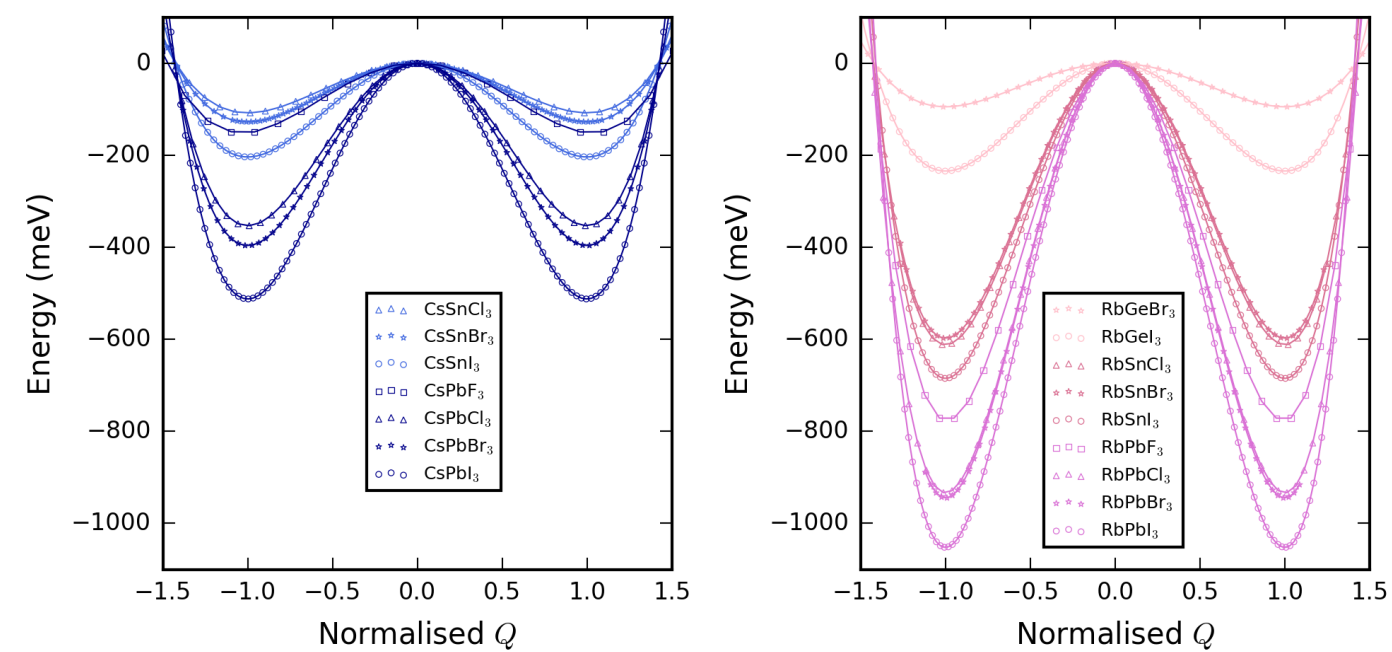

FIG. 2. Double-well potential-energy surfaces for the phonon instabilities associated with $M_{3}^{+}$tilting modes. The left-hand plot shows data for Cs compounds (lines with blue hue), while the right-hand plot shows data for Rb perovskites (lines with red hue). Halide anions are differentiated by the markers shown in the legends. The normal-mode coordinate $Q$ has been normalised so that for each composition the minima are located at $Q=1$, and the energy differences are those calculated in a $2 \times 2 \times 2$ supercell. 
TABLE II. Summary of the imaginary harmonic phonon modes observed for the cubic perovskites. The labels refer to the irreducible representation, ${ }^{24}$ and are illustrated in Fig $1 b$.

\begin{tabular}{ccccc}
\hline \hline Composition & \multicolumn{4}{c}{ Imaginary Mode } \\
& $\Gamma$ & $X$ & $M$ & $R$ \\
\hline $\mathrm{CsGeF}_{3}$ & $\Gamma_{4}^{-}$ & $X_{5}^{-}$ & $M_{2}^{-}$ & \\
$\mathrm{CsGeCl}_{3}$ & $\Gamma_{4}^{-}$ & $X_{5}^{-}$ & $M_{2}^{-}$ & \\
$\mathrm{CsGeBr}_{3}$ & $\Gamma_{4}^{-}$ & $X_{5}^{-}$ & $M_{2}^{-}$ & \\
$\mathrm{CsGeI}_{3}$ & $\Gamma_{4}^{-}$ & $X_{5}^{-}$ & $M_{2}^{-}, M_{3}^{+}$ & $R_{4}^{+}$ \\
$\mathrm{CsSnF}_{3}$ & $\Gamma_{4}^{-}$ & $X_{5}^{-}$ & $M_{2}^{-}$ & $R_{4}^{+}$ \\
$\mathrm{CsSnCl}_{3}$ & $\Gamma_{4}^{-}$ & & $M_{3}^{+}$ & $R_{4}^{+}$ \\
$\mathrm{CsSnBr}_{3}$ & & & $M_{3}^{+}$ & $R_{4}^{+}$ \\
$\mathrm{CsSnI}_{3}$ & $\Gamma_{4}^{-}$ & $X_{4}^{+}$ & $M_{3}^{+}$ & $R_{4}^{+}$ \\
$\mathrm{CsPbF}_{3}$ & $\Gamma_{4}^{-}$ & & $M_{3}^{+}$ & $R_{4}^{+}$ \\
$\mathrm{CsPbCl}_{3}$ & $\Gamma_{4}^{-}$ & $X_{4}^{+}$ & $M_{3}^{+}$ & $R_{4}^{+}$ \\
$\mathrm{CsPbBr}_{3}$ & $\Gamma_{4}^{-}$ & $X_{4}^{+}$ & $M_{3}^{+}$ & $R_{4}^{+}$ \\
$\mathrm{CsPbI}_{3}$ & $\Gamma_{4}^{-}$ & $X_{4}^{+}$ & $M_{3}^{+}$ & $R_{4}^{+}$ \\
$\mathrm{RbGeF}_{3}$ & $\Gamma_{4}^{-}$ & $X_{5}^{-}$ & $M_{2}^{-}, M_{3}^{+}$ & $R_{4}^{+}$ \\
$\mathrm{RbGeCl}_{3}$ & $\Gamma_{4}^{-}$ & $X_{5}^{-}$ & $M_{2}^{-} M_{3}^{+}$ & $R_{4}^{+}$ \\
$\mathrm{RbGeBr}_{3}$ & $\Gamma_{4}^{-}$ & $X_{5}^{-}$ & $M_{3}^{+}$ & $R_{4}^{+}$ \\
$\mathrm{RbGeI}_{3}$ & $\Gamma_{4}^{-}$ & $X_{5}^{-}$ & $M_{3}^{+}$ & $R_{4}^{+}$ \\
$\mathrm{RbSnF}_{3}$ & $\Gamma_{4}^{-}$ & $X_{5}^{-}$ & $M_{2}^{-}$ & $R_{4}^{+}$ \\
$\mathrm{RbSnCl}_{3}$ & $\Gamma_{4}^{-}$ & $X_{4}^{+}$ & $M_{3}^{+}$ & $R_{4}^{+}$ \\
$\mathrm{RbSnBr}_{3}$ & $\Gamma_{4}^{-}$ & $X_{4}^{+}$ & $M_{3}^{+}$ & $R_{4}^{+}$ \\
$\mathrm{RbSnI}_{3}$ & $\Gamma_{4}^{-}$ & $X_{4}^{+}$ & $M_{3}^{+}$ & $R_{4}^{+}$ \\
$\mathrm{RbPbF}_{3}$ & $\Gamma_{4}^{-}$ & $X_{4}^{+}$ & $M_{3}^{+}$ & $R_{4}^{+}$ \\
$\mathrm{RbPbCl}_{3}$ & $\Gamma_{4}^{-}$ & $X_{4}^{+}$ & $M_{3}^{+}$ & $R_{4}^{+}$ \\
$\mathrm{RbPbBr}_{3}$ & $\Gamma_{4}^{-}$ & $X_{4}^{+}$ & $M_{3}^{+}$ & $R_{4}^{+}$ \\
$\mathrm{RbPbI}_{3}$ & $\Gamma_{4}^{-}$ & $X_{4}^{+}$ & $M_{3}^{+}$ & $R_{4}^{+}$ \\
\hline \hline
\end{tabular}
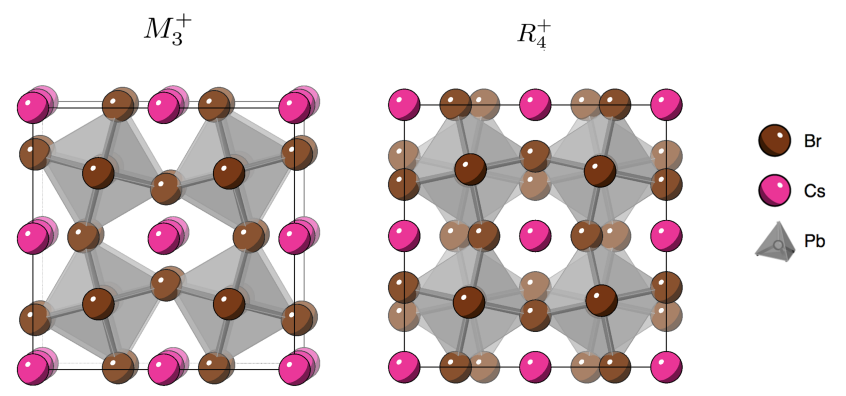

FIG. 3. Distortion of the cubic perovskite structure by condensation of the $M_{3}^{+}$and $R_{4}^{+}$tilting modes in $\mathrm{CsPbBr}_{3}$. The $M_{3}^{+}$and $R_{4}^{+}$modes correspond to in-phase and out-of-phase tilting in successive layers, respectively.

from the centre of the octahedron.

All of these compounds have large tolerance factors ranging from 0.95 to 1.1 , meaning there is limited space for the octahedra to rotate. Instead, the electronic configuration of the B-site cation plays a key role. The electronic configuration $n \mathrm{~s}^{2} n \mathrm{p}^{0}$ for heavy group 14 elements $(\mathrm{Ge}, \mathrm{Sn}$ and $\mathrm{Pb})$ is commonly associated with distorted and non-centrosymmetric coordination environments. The valence $p$ orbitals of the halide mix with the cation $s$ and $p$ orbitals, resulting in a asymmetric

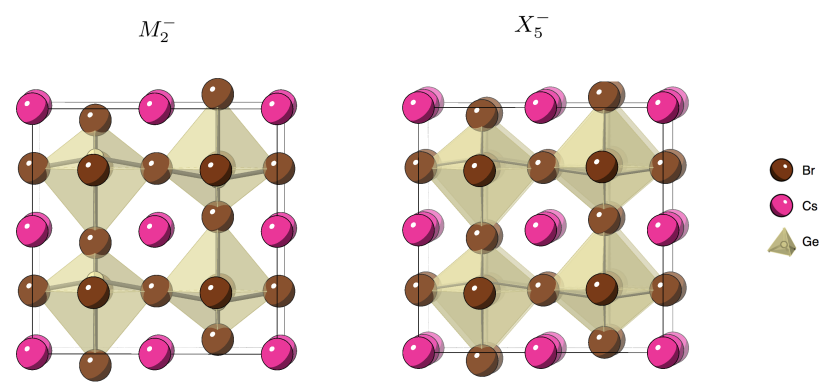

FIG. 4. Zone-boundary Jahn-Teller distortions in $\mathrm{CsGeBr}_{3}$. Shortening and lengthening of the $\mathrm{B}-\mathrm{X}$ bonds results in an off-centering of the Ge cation. The corresponding phonon modes have $M_{2}^{-}$or $X_{5}^{-}$irreducible representations. The images show a $2 \times 2 \times 1$ supercell of the cubic phase in which the two instabilities appear equivalent; the difference is in the movement of atoms in neighbouring supercells in the out-ofplane direction, which is out of phase in the $M_{2}^{-}$mode but in phase in the $X_{5}^{-}$mode.

electron density. These $\mathrm{s}^{2}$ lone pairs can exhibit a static or dynamic structural distortion. ${ }^{55}$ As the binding energy of the $n \mathrm{~s}^{2}$ orbitals increases down the group (i.e. from Ge to $\mathrm{Pb}$ ), in part due to a relativistic contraction, the stability of the lower oxidation states also increases - the so-called "inert pair" effect. ${ }^{63}$ This explains why JahnTeller distortions are present in most of the germanium and the tin-fluoride perovskites.

\section{Zone-centre cation displacements}

With the exception of $\mathrm{CsSnBr}_{3}$, all the perovskites investigated in this work feature $\Gamma$-point phonon instabilities corresponding to a polar $\Gamma_{4}^{-}$mode. 5 The long-range nature of the $\Gamma$ wavevector means that the displacement can induce a macroscopic polarization. ${ }^{64}$ In traditional ferroelectric perovskites, this behaviour occurs through off-centering of the B-site cation, for example $\mathrm{Ti}$ in $\mathrm{BaTiO}_{3}$ and $\mathrm{PbTiO}_{3}$. In these halide perovskites, both the A-site and B-site cations are susceptible to displacement.

For the Ge-based perovskites $\mathrm{CsGeX}_{3}$ and $\mathrm{RbGeX}_{3}$, this mode involves the off-centring of the Ge cation or movement of the bonded halogens, in agreement with the experimentally-identified polar space group $R 3 m$ adopted by $\mathrm{CsGeCl}_{3}, \mathrm{CsGeBr}_{3}$, and $\mathrm{CsGeI}_{3}$. On the other hand, for $\mathrm{Pb}$-based perovskites, the off-centring is most pronounced at the $\mathrm{A}$ site (i.e. $\mathrm{Cs}$ or $\mathrm{Rb}$ are displaced), while $\mathrm{Pb}$ remains at the centre of the octahedron.

For Sn-based compounds, the displacement depends on the halide anions. For $\mathrm{CsSnF}_{3}$, the largest components of the $\Gamma_{4}^{-}$modes are attributed to $\mathrm{F}$, with minor contributions from Sn and Cs. This is due to the light mass of fluorine. Heavier halogens induce relatively more motion of the A-site and B-site cations. 


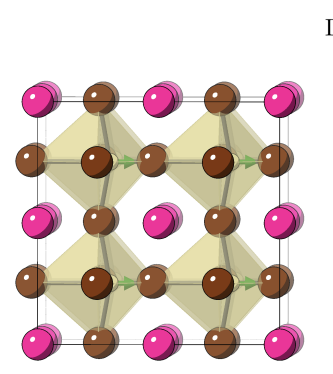

$\Gamma_{4}^{-}$
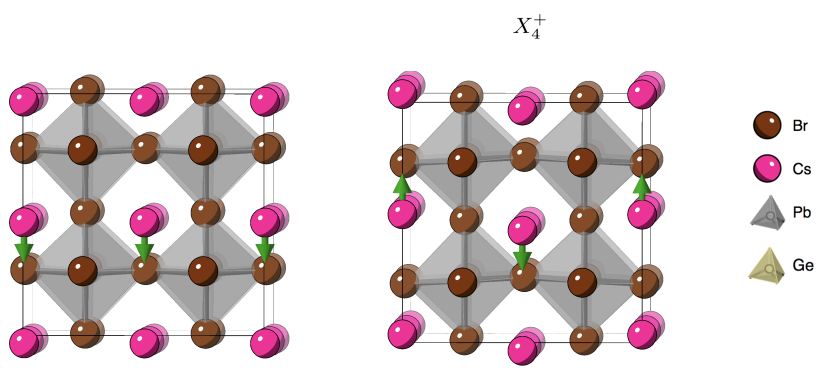

FIG. 5. Change in crystal structure following the $\Gamma_{4}^{-}$mode in both $\mathrm{CsGeBr}_{3}$ and $\mathrm{CsPbBr}_{3}$ and the $X_{5}^{+}$mode in $\mathrm{CsPbBr}_{3}$. For $\mathrm{CsGeBr}_{3}$, the $\Gamma_{4}^{-}$mode corresponds to a B-site displacement, whereas in $\mathrm{CsPbBr}_{3}$ the same mode corresponds to an A-site displacement. For $\mathrm{CsPbBr}_{3}$, the $X_{5}^{+}$mode also corresponds to an A-site displacement, but with out-of-phase motion in neighbouring unit cells.
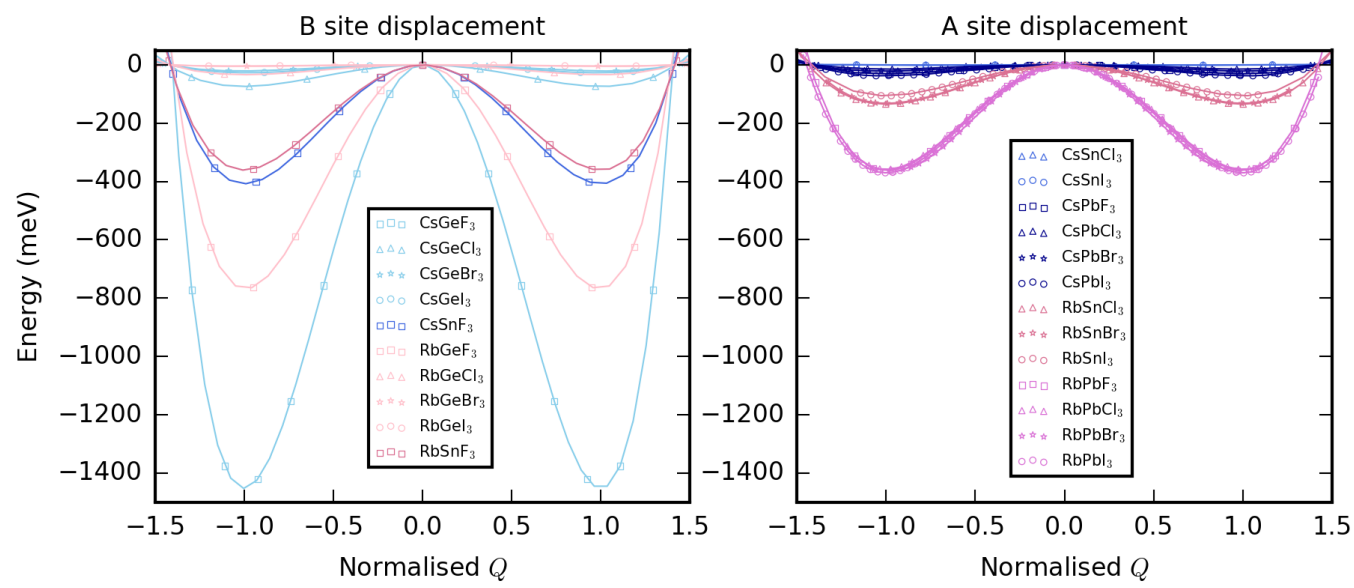

FIG. 6. Comparison of the potential-energy surface in the $\mathrm{ABX}_{3}$ halide perovskites with the polar $\Gamma_{4}^{-}$instabilities. The $\Gamma_{4}^{-}$ instabilities are categorized into B-site displacement and A-site displacement. Lines with blue hue and red hue corresponds to $\mathrm{Cs}$ and $\mathrm{Rb}$ compounds, respectively, and the halide anions are indicated by different markers. The normal-mode coordinate $Q$ has been normalised so that the minima of the wells are located at $Q=1$.

It is worth noting that the $\Gamma$-point modes involving mostly the $\mathrm{Cs}$ or $\mathrm{Rb}$ atoms are relatively flat across reciprocal space, characteristic of decorrelated rattling that would not generate macroscopic polarization. This explains why $\mathrm{Pb}$ and Sn-based perovskites have not been identified in polar space groups.

The $X$-mode instabilities also involve cation displacement. The $X_{4}^{+}$distortion involves motion of the A-site cation (5), whereas the $X_{5}^{-}$mode leads to displacement of the B-site cation. Similar chemical trends as observed for the $\Gamma$-point instabilities can also be seen in the $X$ modes. $\mathrm{CsGeX}_{3}, \mathrm{RbGeX}_{3}, \mathrm{CsSnF}_{3}$ and $\mathrm{RbSnF}_{3}$ show $X_{5}^{-}$instabilities that result in B-site off centring, whereas the other $\mathrm{Sn}$-based and all the $\mathrm{Pb}$-based perovskites undergo $X_{4}^{+}$distortions leading to A site displacement. The key difference between $X$ - and $\Gamma$-point phonon instabilities is that the former involves out-of-phase displacement in neighbouring unit cells along one real-space direction.

To compare the energy landscape associated with the different cation displacements, we mapped out the potential energy surfaces for representative $\Gamma_{4}^{-}$modes (Fig.6).
For the A-site displacement, it is obvious that the double well is deeper for $\mathrm{Rb}$ compounds than Cs compounds (e.g. $\mathrm{CsPbX}_{3}$ vs. $\mathrm{RbPbX}_{3}$ ), which can be explained by the relatively small ionic radius of $\mathrm{Rb}(1.72 \AA$, tolerance factor: $0.82-0.86$ for $\mathrm{RbPbX}_{3}$ ). In both the $\mathrm{Cs}$ and $\mathrm{Rb}$ series, changing the B-site cation from $\mathrm{Sn}$ to $\mathrm{Pb}$ results in a deeper double well potential.

On the other hand, the difference between $\mathrm{Cs}$ and $\mathrm{Rb}$ compounds is not as large for B-site displacement but depends much more strongly on the halide. For the $\mathrm{CsGeX}_{3}$ and $\mathrm{RbGeX}_{3}$ series $(\mathrm{X}=\mathrm{Cl}, \mathrm{Br}, \mathrm{I})$, and for $\mathrm{CsGeF}_{3}$, $\mathrm{RbGeF}_{3}, \mathrm{CsSnF}_{3}$ and $\mathrm{RbSnF}_{3}$, the B-site instabilities are associated with deep energetic minima. For the latter four compounds, the $\Gamma_{4}^{-}$instabilities are accompanied by large distortions to the cage, which results in a correspondingly large reduction in energy. This is consistent with the fact that none of these systems have been experimentally identified. The trend in the B-site displacement indicates that the displacement of the B-site cation within the octahedra is largely independent of the A-site cation, but is affected by the bonding environment 
within the octahedra.

Except for the anomalous fluoride compounds, the energy minima associated with cation displacement are much shallower than those for octahedral tilting, suggesting it to be a secondary effect in reducing the energy.

\section{DISCUSSION AND CONCLUSIONS}

From a bonding perspective, a larger tolerance factor $\alpha$ implies that A-site cation sits in a relatively tight bonding environment and the octahedral network is less prone to tilting. This is evident when the potential-energy minima along the tilt modes are plotted against tolerance factor (Fig. 7). As $\alpha$ approaches unity, the well depths associated with the tilting modes $\left(M_{3}^{+}\right.$and $\left.R_{4}^{+}\right)$become shallower, suggesting weaker tilting instabilities. There is no obvious difference between $M_{3}^{+}$and $R_{4}^{+}$modes, implying that in-phase and out-of-phase tilting are similarly favoured energetically.

Such trends, however, are not present for the zoneboundary Jahn-Teller distortion (Fig. 7), suggesting that they are not a product of the geometry alone. The distortions occur in compound with $\alpha>$ than 0.9 , whereas the tilting mode is more pronounced for compounds with $\alpha<0.9$. This indicates that the JT mode is favoured in relatively small octahedra, which is usually associated with smaller B-site cations and halogen atoms. Also, our calculations show little energetic difference between $M_{2}^{-}$ and $X_{5}^{-}$modes, the two JT-related instabilities.

For cation displacement, there is a distinction between A- and B-site behavior. A clear boundary could be drawn at $\alpha \approx 0.9$, below which $\mathrm{A}$-site displacement is energetically favoured and above which off-centring of the B-site cation is favoured. While both are driven by the same JT distortions, A-site displacement can only be accommodated when the cuboctahedral cavity is large enough, which explains the relation to the tolerance factor. The energy gain (well depths) for cation displacements are generally much smaller than the for other two types of distortions.

At high temperature, these perovskites fluctuate between equivalent structural minima. The depth of the well and the temperature determine the classical hopping rate between two local minima through the Arrhenius equation $\Gamma=\nu \exp \left(-\Delta E / k_{B} T\right)$, where $\nu$ is the attempt frequency. ${ }^{29}$ At high temperature, the compositions with small energy barriers have fast hopping rates and display dynamic disorder, which leads to a cubic symmetry in diffraction on average. On the other hand, those with larger energy barriers show static disorder with slow hopping rates.

Deeper wells lead to a higher phase transition temperature to the cubic phase. This is confirmed by the correlation between the calculated well depths and the measured phase transition temperature (Table I). Although it is not possible to predict the precise phase transition temperature with the single-mode potential sur- faces - this would require high-order anharmonic phonon calculations - it provides a qualitative trend. The distortion modes identified in the high-temperature cubic phase start to condense when the temperature decreases, i.e. the structures start to follow the distortion vectors to lower the energy of the system. For example, the tetragonal phase results from the $M_{3}^{+}$in-phase tilting mode, whereas the orthorhombic Pnma phase results from a combination of $M_{3}^{+}$and $R_{4}^{+}$out-of-phase tilting modes. ${ }^{24}$

We note that all compositions have these phonon modes, and that the modes are highly anharmonic they coexist in perovskite crystals and may couple with each other. Here we have treated each mode independently while "locking in" the other modes. The interaction of different modes requires an explicit treatment of anharmonicity ${ }^{65,66}$ or large-scale molecular dynamics simulations, which is an interesting direction for future research.

In conclusion, we have carried out a systematic study of the phonon instabilities in inorganic halide perovskites. By explicitly mapping the potential-energy surfaces associated with the three classes of instability, we have quantified the relation between the structure and chemistry and the dominant phonon instabilities. All cubic perovskites show octahedral-tilting instabilities, but the magnitude of the tilting and the associated energetic gains depend on the interplay of the A-site cation and the octahedral cage. The JT distortion and B-site displacement both tend to occur in Ge and fluorine compounds due to $s / p$ orbital interactions. A-site cation displacement is favoured when the cation is much smaller than the cage, but results in a moderate energy gain compared to other instabilities. Amongst all instabilities, the octahedral tilting is energetically the most dominant instability. Therefore, our categorization and quantification of the instabilities is especially important in directing the synthesis of new functional inorganic halide perovskites.

\section{ACKNOWLEDGMENTS}

We acknowledge useful discussions with Simon Billinge and Patrick Woodward. Via our membership of the UK's HEC Materials Chemistry Consortium, which is funded by EPSRC (EP/L000202), this work used the ARCHER UK National Supercomputing Service (http://www.archer.ac.uk). This research was also supported by the Creative Materials Discovery Program through the National Research Foundation of Korea (NRF) funded by Ministry of Science and ICT (2018M3D1A1058536). R.X.Y. was funded by ERC Starting Grant no. 277757. J.M.S. is grateful to the EPSRC for funding (grant no. EP/P007821/1) and to the University of Manchester for the award of a Presidential Fellowship. E.L.d.S. is funded by the European Union Horizon 2020 research and innovation programme under Marie Sklodowska-Curie grant agreement No. 785789COMEX. 

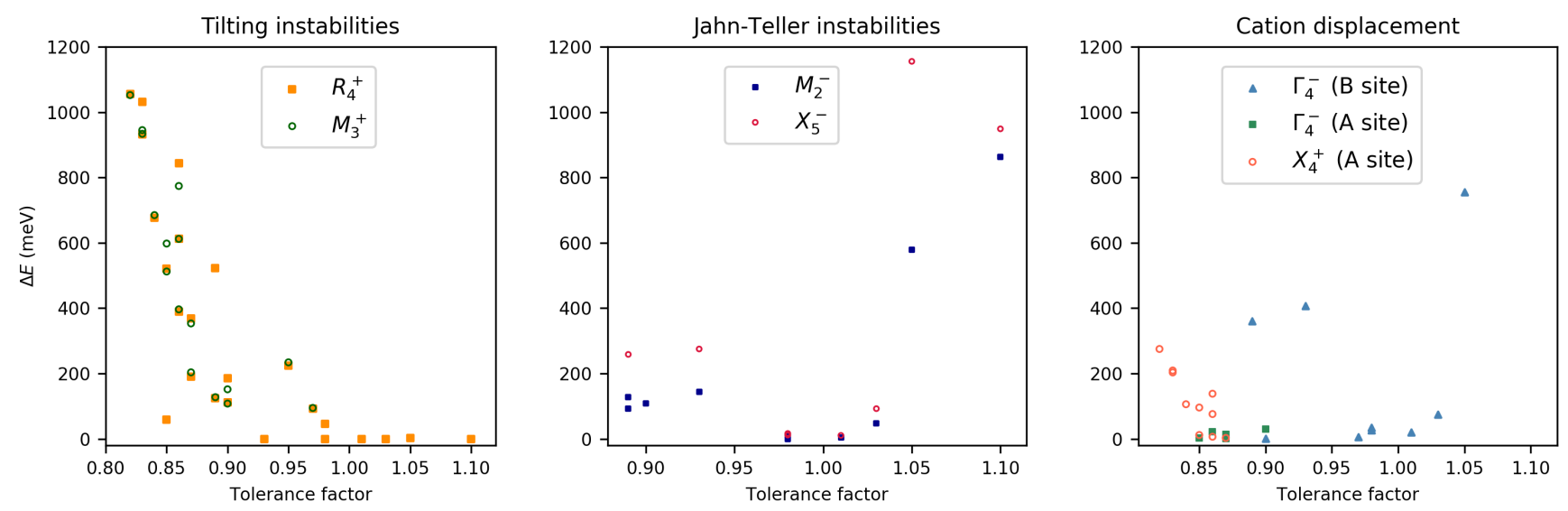

FIG. 7. Relationship between geometric tolerance factor $\alpha$ and well depths associated with different types of phonon instabilities. The tilting instability decreases as tolerance factor increases, while the other two types of instability depend more on the chemistry rather than geometric constraints.

${ }^{1}$ C. K. Møller, Nature 182, 1436 (1958).

${ }^{2}$ J. D. Donaldson, J. Silver, S. Hadjiminolis, and S. D. Ross, J. Chem. Soc., Dalton Trans. 1975, 1500 (1975).

${ }^{3}$ U. Schwarz, H. Hillebrecht, M. Kaupp, K. Syassen, H.-G. von Schnering, and G. Thiele, J. Solid State Chem. 118, 20 (1995).

${ }^{4}$ G. Thiele, H. W. Rotter, and K. D. Schmidt, Zeitschrift für Anorg. und Allg. Chemie 545, 148 (1987).

${ }^{5}$ D. B. Mitzi, C. A. Feild, W. T. A. Harrison, and A. M. Guloy, Nature 369, 467 (1994)

${ }^{6}$ A. Kojima, K. Teshima, Y. Shirai, and T. Miyasaka, J. Am. Chem. Soc. 131, 6050 (2009).

${ }^{7}$ M. M. Lee, J. Teuscher, T. Miyasaka, T. N. Murakami, and H. J. Snaith, Science 338, 643 (2012).

${ }^{8}$ Z. Ning, X. Gong, R. Comin, G. Walters, F. Fan, O. Voznyy, E. Yassitepe, A. Buin, S. Hoogland, and E. H. Sargent, Nature 523, 324 (2015).

${ }^{9}$ C. S. Ponseca, T. J. Savenije, M. a. Abdellah, K. Zheng, A. P. Yartsev, T. Pascher, T. Harlang, P. Chabera, T. Pullerits, A. Stepanov, J.-p. Wolf, and V. Sundstrom, J. Am. Chem. Soc. 136, 5189 (2014).

${ }^{10}$ C. Wehrenfennig, G. E. Eperon, M. B. Johnston, H. J. Snaith, and L. M. Herz, Adv. Mater. 26, 1584 (2014).

${ }^{11}$ Y. Yuan, J. Chae, Y. Shao, Q. Wang, Z. Xiao, A. Centrone, and J. Huang, Adv. Energy. Mater. 5, 1500615 (2015).

${ }^{12}$ S. D. Stranks, G. E. Eperon, G. Grancini, C. Menelaou, M. J. P. Alcocer, T. Leijtens, L. M. Herz, A. Petrozza, and H. J. Snaith, Science 342, 341 (2013).

${ }^{13}$ M. Shirayama, H. Kadowaki, T. Miyadera, T. Sugita, M. Tamakoshi, M. Kato, T. Fujiseki, D. Murata, S. Hara, T. N. Murakami, S. Fujimoto, M. Chikamatsu, and H. Fujiwara, Phys. Rev. Applied 5, 014012 (2016).

${ }^{14}$ D. Weber, Zeitschrift für Naturforsch. 33b, 1443 (1978).

${ }^{15}$ Y. Fujii, S. Hoshino, Y. Yamada, and G. Shirane, Phys. Rev. B 9, 4549 (1974).

${ }^{16}$ R. J. Worhatch, H. J. Kim, I. P. Swainson, A. L. Yonkeu, and S. J. L. Billinge, Chem. Mater. 20, 1272 (2008).

${ }^{17}$ A. N. Beecher, O. E. Semonin, J. M. Skelton, J. M. Frost, M. W. Terban, H. Zhai, A. Alatas, J. S. Owen, A. Walsh, and S. J. L. Billinge, ACS Energy Lett. 1, 880 (2016).

${ }^{18}$ R. Comin, M. K. Crawford, A. H. Said, N. Herron, W. E. Guise, X. Wang, P. S. Whitfield, A. Jain, X. Gong, A. J. H. McGaughey, and E. H. Sargent, Phys. Rev. B 94, 094301 (2016).

${ }^{19}$ K. Page, J. E. Siewenie, P. Quadrelli, and L. Malavasi, Angew. Chemie - Int. Ed. 55, 14320 (2016).
${ }^{20}$ F. Bertolotti, L. Protesescu, M. V. Kovalenko, S. Yakunin, A. Cervellino, S. J. L. Billinge, M. W. Terban, J. S. Pedersen, N. Masciocchi, and A. Guagliardi, ACS Nano 11, 3819 (2017).

${ }^{21}$ O. Yaffe, Y. Guo, L. Z. Tan, D. A. Egger, T. Hull, C. C. Stoumpos, F. Zheng, T. F. Heinz, L. Kronik, M. G. Kanatzidis, J. S. Owen, A. M. Rappe, M. A. Pimenta, and L. E. Brus, Phys. Rev. Lett. 118, 136001 (2017).

${ }^{22}$ J. A. Steele, P. Puech, B. Monserrat, B. Wu, R. X. Yang, T. Kirchartz, H. Yuan, G. Fleury, D. Giovanni, E. Fron, M. Keshavarz, E. Debroye, G. Zhou, T. C. Sum, A. Walsh, J. Hofkens, and M. B. J. Roeffaers, ACS Energy Lett. 4, 2205 (2019), https://doi.org/10.1021/acsenergylett.9b01427.

${ }^{23}$ P. M. Woodward, Acta Crystallogr. Sect. B Struct. Sci. 53, 32 (1997).

${ }^{24}$ C. J. Howard and H. T. Stokes, Acta Crystallogr. Sect. B 54, 782 (1998).

${ }^{25}$ N. A. Benedek and C. J. Fennie, J. Phys. Chem. C 117, 13339 (2013).

${ }^{26}$ J. B. Neaton, C. Ederer, U. V. Waghmare, N. A. Spaldin, and K. M. Rabe, Phys. Rev. B 71, 014113 (2005).

${ }^{27}$ W. J. Merz, Phys. Rev. 76, 1221 (1949).

${ }^{28}$ A. M. Glazer, Acta Crystallogr. Sect. B Struct. Crystallogr. Cryst. Chem. 28, 3384 (1972).

${ }^{29}$ R. X. Yang, J. M. Skelton, E. L. Da Silva, J. M. Frost, and A. Walsh, J. Phys. Chem. Lett. 8, 4720 (2017).

${ }^{30}$ J. K. Burdett, Inorg. Chem. 20, 1959 (1981).

${ }^{31}$ N. A. Benedek and C. J. Fennie, Phys. Rev. Lett. 106, 107204 (2011).

32 J. Young, A. Stroppa, S. Picozzi, and J. Rondinelli, J. Phys. Condens. Matter 27, 283202 (2015).

${ }^{33}$ J. Varignon, N. C. Bristowe, E. Bousquet, and P. Ghosez, Sci. Rep. 5, 15364 (2015).

${ }^{34}$ N. C. Bristowe, J. Varignon, D. Fontaine, E. Bousquet, and P. Ghosez, Nat. Commun. 6, 6677 (2015).

${ }^{35}$ G. Kresse and J. Furthmüller, Phys. Rev. B 54, 11169 (1996).

${ }^{36}$ G. Kresse and J. Furthmüller, Comput. Mater. Sci. 6, 15 (1996).

${ }^{37}$ J. P. Perdew, A. Ruzsinszky, G. I. Csonka, O. A. Vydrov, G. E. Scuseria, L. A. Constantin, X. Zhou, and K. Burke, Phys. Rev. Lett. 100, 136406 (2008).

${ }^{38}$ J. P. Perdew, A. Ruzsinszky, G. I. Csonka, O. A. Vydrov, G. E. Scuseria, L. A. Constantin, X. Zhou, and K. Burke, Phys. Rev. Lett. 102, 39902 (2009), arXiv:0711.0156v3.

${ }^{39}$ G. Bergerhoff, R. Hundt, R. Sievers, and I. D. Brown, J. Chem. Inf. Comput. Sci. 23, 66 (1983). 
${ }^{40}$ J. M. Skelton, L. A. Burton, S. C. Parker, A. Walsh, C.-E. Kim, A. Soon, J. Buckeridge, A. A. Sokol, C. R. A. Catlow, A. Togo, and I. Tanaka, Phys. Rev. Lett. 117, 075502 (2016).

${ }^{41}$ J. M. Skelton, "ModeMap," https://github.com/JMSkelton/ModeMap.

${ }^{42}$ A. N. Christensen and S. E. Rasmussen, ACTA Chem. Scand. 19, 421 (1965).

${ }^{43}$ M. Ahtee, K. Kurki-Suonio, A. Vahvaselkä, A. W. Hewat, J. Harada, and S. Hirotsu, Acta Crystallogr. Sect. B 36, 1023 (1980).

${ }^{44}$ M. Sakata, N. Takash, and H. Jimpei, J. Phys. Soc. Japan 47, 232 (1979).

${ }^{45}$ D. Trots and S. Myagkota, J. Phys. Chem. Solids 69, 2520 (2008).

${ }^{46}$ I. Chung, J. H. Song, J. Im, J. Androulakis, C. D. Malliakas, H. Li, A. J. Freeman, J. T. Kenney, and M. G. Kanatzidis, J. Am. Chem. Soc. 134, 8579 (2012).

${ }^{47}$ G. Thiele, H. W. Rotter, and K. D. Schmidt, Z. Anorg. Allg. Chem. 571, 60 (1989).

${ }^{48}$ Y. Yamane, K. Yamada, and K. Inoue, Solid State Ionics 179, 605 (2008).

${ }^{49}$ V. M. Goldschmidt, Naturwissenschaften 14, 477 (1926).

${ }^{50}$ D.-K. Seo, N. Gupta, M.-H. Whangbo, H. Hillebrecht, and G. Thiele, Inorg. Chem. 37, 407 (1998).

${ }^{51}$ R. D. Shannon, Acta Crystallogr. Sect. A 32, 751 (1976).

${ }^{52}$ G. Kieslich, S. Sun, and T. Cheetham, Chem. Sci. 6, 3430 (2015).

${ }^{53} \mathrm{~K}$. Yamada, K. Isobe, T. Okuda, and Y. Furukawa, Zeitschrift Naturforschung Teil A 49, 258 (1994).
${ }^{54}$ J. Barrett, S. R. A. Bird, J. D. Donaldson, and J. Silver, J. Chem. Soc. A , 3105 (1971).

${ }^{55}$ D. H. Fabini, G. Laurita, J. S. Bechtel, C. C. Stoumpos, H. A. p. Evans, A. G. Kontos, Y. S. Raptis, P. Falaras, A. Van der Ven, M. G. Kanatzidis, and R. Seshadri, J. Am. Chem. Soc. 138, 11820 (2016).

${ }^{56}$ R. Clarke, E. Marseglia, and H. P. Hughes, Philos. Mag. Part B 38, 121 (1978).

${ }^{57}$ P. Berastegui, S. Hull, and S. Eriksson, J. Phys. Condens. Matter 13, 5077 (2001).

${ }^{58}$ S. Hirotsu, J. Harada, M. Iizumi, and K. Gesi, J. Phys. Soc. Japan 37, 1393 (1974).

${ }^{59}$ S. Picozzi, K. Yamauchi, I. A. Sergienko, C. Sen, B. Sanyal, and E. Dagotto, J. Phys. Condens. Matter 20, 434208 (2008).

${ }^{60}$ G.-J. Yang, B. Ding, Y. Li, S.-Y. Huang, Q.-Q. Chu, C. Li, and C. Li, J. Mater. Chem. A 5, 6840.

${ }^{61}$ R. D. King-Smith and D. Vanderbilt, Phys. Rev. B 49, 5828 (1994).

${ }^{62}$ W. Zhong, D. Vanderbilt, and K. M. Rabe, Phys. Rev. B 52, 6301 (1995).

${ }^{63}$ A. Walsh, D. J. Payne, R. G. Egdell, and G. W. Watson, Chem. Soc. Rev. 40, 4455 (2011).

${ }^{64}$ H. T. Stokes, E. H. Kisi, D. M. Hatch, and C. J. Howard, Acta Crystallogr. Sect. B 58, 934 (2002).

${ }^{65}$ N. R. Werthamer, Phys. Rev. B 1, 572 (1970).

${ }^{66}$ I. Errea, M. Calandra, and F. Mauri, Phys. Rev. B 89, 064302 (2014). 